\title{
Optimization of a synthetic medium for ethanol production by xylose-fermenting Zymomonas mobilis using response surface methodology
}

\author{
DONG HuiNa ${ }^{1,2,4,5}$, ZHAO XueMing ${ }^{2,4,5}$, MA YuanYuan ${ }^{1 *} \&$ ZHANG MinHua ${ }^{1,3}$ \\ ${ }^{1}$ Tianjin University R\&D Center for Petrochemical Technology, Tianjin University, Tianjin 300072, China; \\ ${ }^{2}$ Department of Biochemical Engineering, School of Chemical Engineering and Technology, Tianjin University, Tianjin 300072, China; \\ ${ }^{3}$ State Key Laboratory of Engines, Tianjin University, Tianjin 300072, China; \\ ${ }^{4}$ Key Laboratory of Systems Bioengineering, Ministry of Education, Tianjin University, Tianjin 300072, China; \\ ${ }^{5}$ Edinburgh-Tianjin Joint Research Centre for Systems Biology and Synthetic Biology, Tianjin University, Tianjin 300072, China
}

Received October 26, 2011; accepted January 31, 2012; published online August 3, 2012

\begin{abstract}
A synthetic medium for ethanol production by recombinant xylose-fermenting Zymomonas mobilis was optimized using Plackett-Burman (PB) design and response surface methodology (RSM). The effects of 19 medium components were investigated by PB design. Eight of these components were determined to have significant effects on ethanol production. The statistical model was constructed via central composite design (CCD) using five selected variables, including xylose, trisodium citrate, choline chloride, pyridoxine, and thiamine. The validity of the developed model was verified. The ethanol concentration in the optimized medium was $6.7 \%$ higher than in the RM medium. The optimized medium was then simplified to give the final synthetic medium (S2), in which the ethanol concentration was $20.7 \%$ higher than in the RM medium. Xylose and trisodium citrate were the key medium components influencing ethanol production, while calcium pantothenate was the only growth factor required by recombinant Z. mobilis. Ethanol production and growth response were directly proportional to the logarithm of the concentration of trisodium citrate in the range of $0.1-1.6 \mathrm{~g} / \mathrm{L}$.
\end{abstract}

recombinant Zymomonas mobilis, synthetic medium, ethanol production, response surface methodology, trisodium citrate, xylose

Citation: Dong H N, Zhao X M, Ma Y Y, et al. Optimization of a synthetic medium for ethanol production by xylose-fermenting Zymomonas mobilis using response surface methodology. Chin Sci Bull, 2012, 57: 3782-3789, doi: 10.1007/s11434-012-5326-5

Rising concerns over the cost of petroleum and the prospect of global warming are driving researchers to develop alternative fuel production technologies. Ethanol is believed to be one of the best alternatives due to its renewable and environmentally friendly properties $[1,2]$. Among many microorganisms that have been exploited for ethanol production, Saccharomyces cerevisiae still remains the most widely used [1]. However, Zymomonas mobilis has been the object of considerable interest in the past decades because of its ability to ferment hexoses into ethanol rapidly and efficiently [3]. Despite this, wild Z. mobilis strains cannot

\footnotetext{
*Corresponding author (email: myy@tju.edu.cn)
}

use xylose, the major component of hemicellulose [4]. Hence, many efforts have been devoted to develop efficient xylose-fermenting $Z$. mobilis, and at present, several recombinant strains capable of fermenting xylose to ethanol have been engineered [5,6]. However, the ability of engineered strains to metabolize xylose is lower than their ability to metabolize glucose.

Apart from selecting a productive strain, designing an appropriate fermentation medium is of crucial importance to improve the efficiency of ethanol production [7]. Because the synthetic medium is usually composed of known amounts of pure chemicals, it is possible to determine the specific requirements for growth and product formation by 
systematically adding or eliminating components from the formulation, which also results in more stability and reproducible culture conditions [8]. A synthetic medium is thus well suited for fundamental studies of microbe metabolism and fermentation optimization.

Statistical experimental designs such as the PlackettBurman (PB) design [9], steepest ascent method [10] and response surface methodology (RSM) [11] can collectively optimize all the parameters by conducting a minimal number of experiments. These designs are good for screening and optimizing medium compositions and culture conditions in fermentation processes [12-15]. A report described the application of RSM to the optimization of ethanol production in RM medium with glucose as the sole carbon source for $Z$. mobilis, obtaining an ethanol yield of $0.5 \mathrm{~g} / \mathrm{g}$, which was very close to the predicted value $(0.51 \mathrm{~g} / \mathrm{g})$ [16]. RSM was also applied to the optimization of ethanol production from waste cashew apple juice [17], sugar cane molasses [15] and carob pod extract [2]. Although optimal ethanol production by wild Z. mobilis was achieved, these complicated media are not suitable for studies on microbial growth, product formation and metabolism. An appropriate synthetic medium containing xylose is necessary for the conduction of metabolism studies to improve the xylose fermentation capability of recombinant Z. mobilis. However, until now, there has not been a detailed study applying these statistical designs to the optimization of a xylose-containing synthetic medium for ethanol production. Therefore, this work evaluates the effects of medium composition on ethanol production from xylose, and presents the formulation of a synthetic medium for optimum ethanol production by xylose-fermenting Z. mobilis, based on statistical experimental designs.

\section{Materials and methods}

\subsection{Microorganisms}

Xylose-fermenting recombinant Z. mobilis CP4-P2-1 harboring pZ-XXTT2 plasmid were used in this study. The pZ-XXTT2 plasmid contains foreign xylose metabolism genes encoding XI (xylose isomerase), XK (xylulokinase), TAL (transaldolase) and TKT (transketolase). The strain engineered by our laboratory [18] is capable of cofermenting the glucose and xylose present in the $\mathrm{RM}$ medium (20 g/L xylose, $10 \mathrm{~g} / \mathrm{L}$ yeast extract, $2 \mathrm{~g} / \mathrm{L} \mathrm{KH}_{2} \mathrm{PO}_{4}$ and $15 \mathrm{mg} / \mathrm{L}$ tetracycline) to ethanol.

\subsection{Seed culture preparation}

The strain was pre-cultured in $10 \mathrm{~mL}$ screw-cap test tubes containing $5 \mathrm{~mL}$ RM medium until the cell density reached OD 1.0 (optical density, $600 \mathrm{~nm}, 1 \mathrm{~cm}$ light path), and then transferred to a $250 \mathrm{~mL}$ flask containing $100 \mathrm{~mL} \mathrm{RM} \mathrm{me-}$ dium. When the cell density reached 1.0-1.2 OD, a designated volume of seed culture was centrifuged and the cell pellets were resuspended in sterilized water to initiate fermentation.

\subsection{Fermentation medium and culture conditions}

The fermentation medium contained salts, trace elements, vitamins, and growth factors. All chemicals used were of analytical grade. The trace elements, vitamins, and growth factors were all purchased from Beijing Aoboxing Bio-tech Co. Ltd. (Beijing, China). The components and their concentrations were specified according to statistical experimental designs. All the experiments, except for the investigation of the effect of trisodium citrate on ethanol production, were performed in $250 \mathrm{~mL}$ shake flasks containing 60 $\mathrm{mL}$ of fermentation medium, according to the experimental designs. Fermentations were performed at an initial OD600 of approximately 0.1 , at $30^{\circ} \mathrm{C}$ for $96 \mathrm{~h}$, without agitation.

\subsection{Analytical techniques}

The biomass concentration was determined by OD600 using a Varian Cary $50 \mathrm{UV}-\mathrm{Vis}$ spectrophotometer (Palo Alto, CA, USA). Samples for quantitative analysis were centrifuged at $13000 \mathrm{r} / \mathrm{min}$ for $10 \mathrm{~min}$ at $20^{\circ} \mathrm{C}$. The supernatants were filtered through a $0.22 \mu \mathrm{m}$ membrane filter and then used to determine the concentrations of glucose, xylose, ethanol and their by-products (lactic acid, glycerol and acetic acid) with an Agilent 1200 high pressure liquid chromatograph (HPLC; Palo Alto). An Aminex HPX-87H organic acid column (BIO-RAD, Hercules, CA), set at $40^{\circ} \mathrm{C}$, was used to separate the compounds. The mobile phase was $4 \mathrm{mmol} / \mathrm{L}$ $\mathrm{H}_{2} \mathrm{SO}_{4}$, and the flow rate was $0.6 \mathrm{~mL} / \mathrm{min}$.

\subsection{Experimental design and statistical analysis}

(i) Plackett-Burman design. To identify the significant variables that affect ethanol production, different medium components were evaluated applying the Plackett-Burman (PB) experimental design. The total number of trials to be carried out according to $\mathrm{PB}$ is $k+1$, where $k$ is the number of variables. Twenty-three variables, including 19 medium components $\left(A\right.$, xylose; $B,\left(\mathrm{NH}_{4}\right)_{2} \mathrm{SO}_{4} ; C, \mathrm{MgSO}_{4} \cdot 7 \mathrm{H}_{2} \mathrm{O} ; D$, $\mathrm{KH}_{2} \mathrm{PO}_{4} ; F$, trisodium citrate; $G, \mathrm{FeSO}_{4} \cdot 7 \mathrm{H}_{2} \mathrm{O} ; H$, succinic acid; $J$, methionine; $L$, lysine; $M$, biotin; $N$, choline chloride; $O$, cyanocobalamin; $Q$, folic acid; $R$, inositol; $S$, nicotinic acid; $T, p$-aminobenzoic acid; $V$, pyridoxine; $W$, riboflavin; $X$, thiamine) and four dummy variables $(E, K, P, U)$, were screened in 24 trials. The low (-1) and high (1) levels of each ingredient were chosen to encompass the range described in the literature and to reflect what was done in practice after a preliminary investigation of the limits. The experiments were carried out according to the matrix obtained with Design Expert 7.0 (Stat-Ease, Inc., MN, USA) as shown in Table $\mathrm{S} 1$.

(ii) Steepest ascent method. The steepest ascent method 
is an effective experimental approach to move sequentially along the path of steepest ascent towards the optimum, so near-optimum conditions are reached rapidly and efficiently [10]. The path of steepest ascent is the direction in which the response increases most rapidly. This method was used to check whether we were prospecting the best range of the five variables (xylose, trisodium citrate, choline chloride, pyridoxine and thiamine) identified by the PB design. Table 1 shows the experimental design for six trials applying the steepest ascent method. The best combination was selected as the central coded value of zero in the central composite design (CCD).

(iii) Central composite design and response surface methodology. RSM, which includes factorial design and regression analysis, is used for the evaluation of effective factors and for the construction of models to study interactions, selecting the optimum conditions for a desirable response [19]. RSM-CCD built using Design Expert 7.0 was applied to find out the optimum concentrations of the five selected variables described above and to study their interactions. Each variable was analyzed at five different levels, with all variables taken at a central coded value of zero (Table 2). A half-factorial experimental design combining six replicates at the central point and ten axial points $(\alpha=2)$, with a total of 32 experiments, was used. The matrix for this design and the experimental response values (ethanol concentration) are shown in Table 3.

\section{Results}

\subsection{Screening of significant variables using PB design}

Pre-screening experiments showed that the strains in the synthetic medium lacking calcium pantothenate did not grow at all. This indicates that calcium pantothenate is a necessary growth factor for recombinant $Z$. mobilis. Thus, calcium pantothenate was added to the synthetic medium at a concentration of $2.74 \mathrm{mg} / \mathrm{L}$, according to De Graaf et al. [20]. The significance of each variable was determined by the method of Wu et al. [12] on the basis of ethanol production (Table S1). Variables with confidence levels above 95\% $(P<0.05)$ were considered to have significant effects. Based on the statistical analysis (Figure 1), the factors having significant effects on ethanol production were identified as $A$ (xylose), $B\left(\left(\mathrm{NH}_{4}\right)_{2} \mathrm{SO}_{4}\right), F$ (trisodium citrate), $H$ (suc- cinic acid), $N$ (choline chloride), $Q$ (folic acid), $V$ (pyridoxine) and $X$ (thiamine). Moreover, $B\left(\left(\mathrm{NH}_{4}\right)_{2} \mathrm{SO}_{4}\right), D$ $\left(\mathrm{KH}_{2} \mathrm{PO}_{4}\right), H$ (succinic acid), $G\left(\mathrm{FeSO}_{4} \cdot 7 \mathrm{H}_{2} \mathrm{O}\right), L$ (lysine), $Q$ (folic acid), $S$ (nicotinic acid) and $W$ (riboflavin) were set at their low levels due to their negative effects. Although the effects of $C\left(\mathrm{MgSO}_{4} \cdot 7 \mathrm{H}_{2} \mathrm{O}\right), J$ (methionine), $M$ (biotin), $O$ (cyanocobalamin), $R$ (inositol) and $T$ ( $p$-aminobenzoic acid) on ethanol production were insignificant, they were chosen at their high levels because of their positive effects.

Five significant components (xylose, trisodium citrate, choline chloride, pyridoxine and thiamine) were selected for the subsequent experiments. The effects of these five variables on ethanol production were greater at higher concentrations. The concentrations of the rest of the variables in the optimal medium were set as follows: $1 \mathrm{~g} / \mathrm{L}\left(\mathrm{NH}_{4}\right)_{2} \mathrm{SO}_{4}, 1$ $\mathrm{g} / \mathrm{L} \mathrm{MgSO}_{4} \cdot 7 \mathrm{H}_{2} \mathrm{O}, 2 \mathrm{~g} / \mathrm{L} \mathrm{KH}_{2} \mathrm{PO}_{4}, 0.1 \mathrm{~g} / \mathrm{L}$ methionine, 33 $\mu \mathrm{g} / \mathrm{L}$ biotin, $1 \mu \mathrm{g} / \mathrm{L}$ cyanocobalamin, $0.14 \mathrm{mg} / \mathrm{L}$ inositol, $7.64 \mathrm{mg} / \mathrm{L} p$-aminobenzoic acid.

\subsection{Steepest ascent method}

The coefficients obtained by PB design offered the directions and steps for the steepest ascent method. An increase in the response was observed in the second step (Table 1). However, all further steps resulted in a significant decrease in ethanol production. This indicates that the concentrations of the medium components should be close to those used in the second step $(70 \mathrm{~g} / \mathrm{L}$ xylose, $2.6 \mathrm{~g} / \mathrm{L}$ trisodium citrate, 6 $\mathrm{mg} / \mathrm{L}$ choline chloride, $0.86 \mathrm{mg} / \mathrm{L}$ pyridoxine and 1.06 $\mathrm{mg} / \mathrm{L}$ thiamine).

\subsection{Response surface methodology}

The concentrations of the aforementioned five components were optimized to find the best combination of fermentation parameters for maximum ethanol production. The experimental data in Table 3 were correlated as a second order polynomial model by nonlinear regression. The empirical relationship between variables and responses was expressed by the following second-order polynomial equation:

$$
\begin{aligned}
Y= & +17.76-3.20 A+0.34 B-0.14 C-0.20 D+0.23 E \\
& -0.26 A B-0.42 A C-0.20 A D-0.21 A E-0.37 B C \\
& +0.059 B D+0.089 B E+0.043 C D-0.59 C E-0.34 D E \\
& -4.41 A^{2}-2.45 B^{2}-0.20 C^{2}-0.077 D^{2}-0.046 E^{2},
\end{aligned}
$$

\begin{tabular}{|c|c|c|c|c|c|c|}
\hline Step & Xylose (g/L) & Trisodium citrate $(\mathrm{g} / \mathrm{L})$ & Choline chloride $(\mathrm{mg} / \mathrm{L})$ & Pyridoxine (mg/L) & Thiamine $(\mathrm{mg} / \mathrm{L})$ & 96 h, Ethanol (g/L), $n=2$ \\
\hline 0 & 10 & 0.2 & 0 & 0 & 0 & $3.08 \pm 0.23$ \\
\hline 1 & 40 & 1.4 & 3 & 0.43 & 0.53 & $15.16 \pm 0.49$ \\
\hline 2 & 70 & 2.6 & 6 & 0.86 & 1.06 & $19.47 \pm 0.37$ \\
\hline 3 & 100 & 3.8 & 9 & 1.29 & 1.59 & $5.94 \pm 0.16$ \\
\hline 4 & 130 & 5.0 & 12 & 1.72 & 2.12 & $0.30 \pm 0.11$ \\
\hline
\end{tabular}

Table 1 Design and results of the steepest ascent experiments 
Table 2 Variables and levels used for the central composite design ${ }^{\text {a) }}$

\begin{tabular}{|c|c|c|c|c|c|c|c|}
\hline \multirow{2}{*}{ Variables } & \multicolumn{2}{|c|}{ Symbols } & \multicolumn{5}{|c|}{ Levels } \\
\hline & Uncoded & Coded & -2 & -1 & 0 & 1 & 2 \\
\hline Xylose (g/L) & $x_{1}$ & $A$ & 10 & 40 & 70 & 1001 & 130 \\
\hline Trisodium citrate $(\mathrm{g} / \mathrm{L})$ & $x_{2}$ & $B$ & 0.2 & 1.4 & 2.6 & 3.8 & 5.0 \\
\hline Choline chloride (mg/L) & $x_{3}$ & $C$ & 0 & 6 & 12 & 18 & 24 \\
\hline Pyridoxine (mg/L) & $x_{4}$ & $D$ & 0 & 0.86 & 1.72 & 2.58 & 3.44 \\
\hline Thiamine (mg/L) & $x_{5}$ & $E$ & 0 & 1.06 & 2.12 & 3.18 & 4.24 \\
\hline
\end{tabular}

Table 3 Experimental design and results of CCD

\begin{tabular}{|c|c|c|c|c|c|c|}
\hline Run & $A$ & $B$ & $C$ & $D$ & $E$ & 96 h, Ethanol $(\mathrm{g} / \mathrm{L})$ \\
\hline 1 & -1 & -1 & -1 & -1 & 1 & 14.81 \\
\hline 2 & 1 & -1 & -1 & -1 & -1 & 5.93 \\
\hline 3 & -1 & 1 & -1 & -1 & -1 & 13.29 \\
\hline 4 & 1 & 1 & -1 & -1 & 1 & 7.19 \\
\hline 5 & -1 & -1 & 1 & -1 & -1 & 15.19 \\
\hline 6 & 1 & -1 & 1 & -1 & 1 & 5.74 \\
\hline 7 & -1 & 1 & 1 & -1 & 1 & 14.50 \\
\hline 8 & 1 & 1 & 1 & -1 & -1 & 4.93 \\
\hline 9 & -1 & -1 & -1 & 1 & -1 & 12.83 \\
\hline 10 & 1 & -1 & -1 & 1 & 1 & 5.29 \\
\hline 11 & -1 & 1 & -1 & 1 & 1 & 14.88 \\
\hline 12 & 1 & 1 & -1 & 1 & -1 & 5.16 \\
\hline 13 & -1 & -1 & 1 & 1 & 1 & 13.98 \\
\hline 14 & 1 & -1 & 1 & 1 & -1 & 6.37 \\
\hline 15 & -1 & 1 & 1 & 1 & -1 & 14.95 \\
\hline 16 & 1 & 1 & 1 & 1 & 1 & 2.68 \\
\hline 17 & -2 & 0 & 0 & 0 & 0 & 2.94 \\
\hline 18 & 2 & 0 & 0 & 0 & 0 & 0.11 \\
\hline 19 & 0 & -2 & 0 & 0 & 0 & 6.66 \\
\hline 20 & 0 & 2 & 0 & 0 & 0 & 12.08 \\
\hline 21 & 0 & 0 & -2 & 0 & 0 & 18.94 \\
\hline 22 & 0 & 0 & 2 & 0 & 0 & 17.78 \\
\hline 23 & 0 & 0 & 0 & -2 & 0 & 18.73 \\
\hline 24 & 0 & 0 & 0 & 2 & 0 & 19.02 \\
\hline 25 & 0 & 0 & 0 & 0 & -2 & 17.72 \\
\hline 26 & 0 & 0 & 0 & 0 & 2 & 20.27 \\
\hline 27 & 0 & 0 & 0 & 0 & 0 & 17.49 \\
\hline 28 & 0 & 0 & 0 & 0 & 0 & 18.38 \\
\hline 29 & 0 & 0 & 0 & 0 & 0 & 17.01 \\
\hline 30 & 0 & 0 & 0 & 0 & 0 & 18.68 \\
\hline 31 & 0 & 0 & 0 & 0 & 0 & 15.78 \\
\hline 32 & 0 & 0 & 0 & 0 & 0 & 16.38 \\
\hline
\end{tabular}

where $Y$ is the predicted response of ethanol production $(\mathrm{g} / \mathrm{L})$ and $A, B, C, D, E$ are the coded values of xylose, trisodium citrate, choline chloride, pyridoxine and thiamine, respectively.

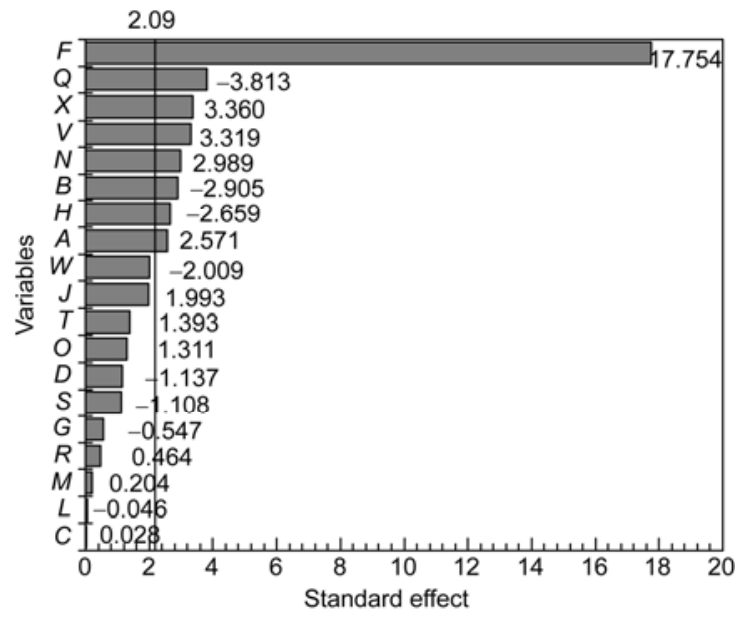

Figure 1 Effects of the tested variables on ethanol production, determined by PB design. Ethanol production is the response $(\alpha=0.05)$.

The sufficiency of the second-order model was checked by the analysis of variance (ANOVA) presented in Table 4. The $F$-value of 4.28 implies that the model is significant, as there is only a $0.84 \%$ probability that a large $F$-value could occur due to noise. The model presents a relatively high determination coefficient $\left(R^{2}=0.886\right)$, explaining $88.6 \%$ of the variability in the response. An $R^{2}$ value higher than 0.80 indicates that the model fits the experimental data reasonably well. The $P$-values were used to identify the effects of each factor on ethanol production. A $P$-value below 0.05 indicates that the model terms are significant. In this case, $A$ (xylose), $A^{2}$ (xylose $\times$ xylose) and $B^{2}$ (trisodium citrate $\times$ trisodium citrate) are significant (Table 5).

Three-dimensional response surface curves were plotted to study the interaction between the two factors selected and determine their optimum concentrations for maximum ethanol production. Figure 2 shows the relationship between xylose and trisodium citrate, while keeping the other three nutrients at constant levels. The marked convexity of the response surface suggests that the optimum conditions are well defined. The circular nature of the contour plot in dicates that the interaction between xylose and trisodium citrate is negligible. The location of the optimum was determined to be $A=-0.382, B=0.046, C=0.525, D=0.107$ and $E=-0.351$, obtained by the differentiation of the

Table 4 ANOVA of the quadratic model ${ }^{\mathrm{a})}$

\begin{tabular}{crrrrr}
\hline Source & \multicolumn{1}{c}{ SS } & DF & \multicolumn{1}{c}{ MS } & $F$-value & $P$-value \\
\hline Model & 980.28 & 20 & 49.01 & 4.28 & 0.0084 \\
Residual & 125.86 & 11 & 11.44 & & \\
Lack of fit & 119.51 & 6 & 19.92 & 15.69 & 0.0042 \\
Pure error & 6.35 & 5 & 1.27 & & \\
Cor total & 1106.13 & 31 & & & \\
\hline
\end{tabular}

a) $R^{2}=0.8862 ; \mathrm{SS}$, sum of squares; $\mathrm{DF}$, degrees of freedom; MS, mean square; $\operatorname{Adj} R^{2}=0.6793$. 
Table 5 Regression coefficients and significances obtained by the response surface model

\begin{tabular}{cccr}
\hline Factor & Coefficient estimate & $F$-value & $P$-value \\
\hline Intercept & 17.7632 & & \\
$A$-Xylose & -3.2001 & 21.4695 & 0.0007 \\
$B$-Trisodium citrate & 0.3430 & 0.2467 & 0.6292 \\
$C$-Choline chloride & -0.1397 & 0.0409 & 0.8434 \\
$D$-Pyridoxine & -0.2022 & 0.0857 & 0.7752 \\
$E$-Thiamine & 0.2299 & 0.1108 & 0.7455 \\
$A B$ & -0.2608 & 0.0950 & 0.7636 \\
$A C$ & -0.4152 & 0.2409 & 0.6332 \\
$A D$ & -0.1967 & 0.0541 & 0.8204 \\
$A E$ & -0.2128 & 0.0633 & 0.8060 \\
$B C$ & -0.3675 & 0.1887 & 0.6724 \\
$B D$ & 0.0592 & 0.0049 & 0.9455 \\
$B E$ & 0.0890 & 0.0111 & 0.9181 \\
$C D$ & 0.0426 & 0.0025 & 0.9607 \\
$C E$ & -0.5936 & 0.4925 & 0.4974 \\
$D E$ & -0.3371 & 0.1588 & 0.6979 \\
$A^{2}$ & -4.4142 & 49.9298 & $<0.0001$ \\
$B^{2}$ & -2.4539 & 15.4300 & 0.0024 \\
$C^{2}$ & -0.2050 & 0.1077 & 0.7490 \\
$D^{2}$ & -0.0771 & 0.0152 & 0.9040 \\
$E^{2}$ & -0.0463 & 0.0055 & 0.9423 \\
\hline
\end{tabular}

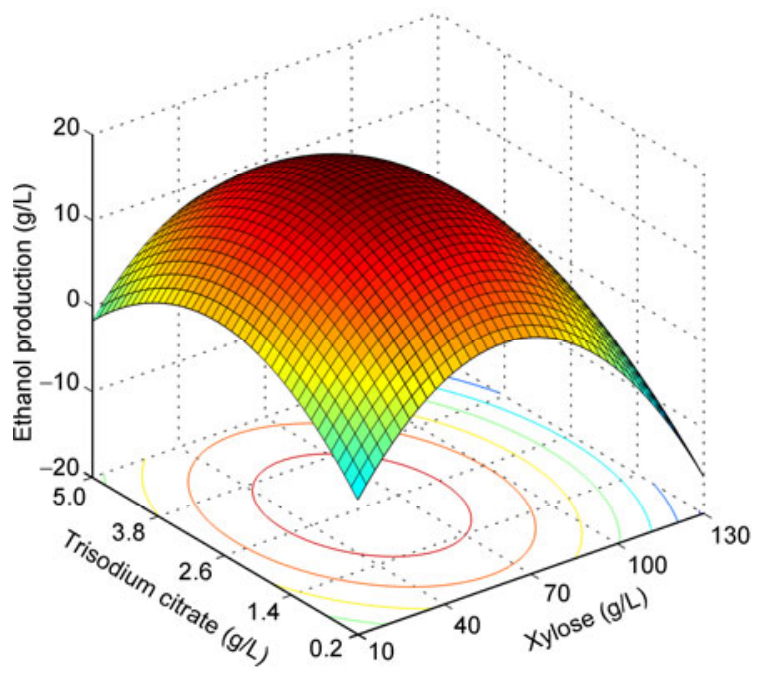

Figure 2 Effect of the interaction between xylose and trisodium citrate on ethanol production. Actual factors: $4.35 \mathrm{mg} / \mathrm{L}$ choline chloride, 1.81 $\mathrm{mg} / \mathrm{L}$ pyridoxine, $1.75 \mathrm{mg} / \mathrm{L}$ thiamine.

quadratic model given by eq. (1). The corresponding uncoded values were: $58.5 \mathrm{~g} / \mathrm{L}$ xylose, $2.65 \mathrm{~g} / \mathrm{L}$ trisodium citrate, $4.35 \mathrm{mg} / \mathrm{L}$ choline chloride, $1.81 \mathrm{mg} / \mathrm{L}$ pyridoxine and $1.75 \mathrm{mg} / \mathrm{L}$ thiamine. The predicted optimal ethanol production according to these values was $18.3 \mathrm{~g} / \mathrm{L}$.

\subsection{Verification by batch culture in shake flasks}

To confirm the adequacy of the model to predict maximum ethanol production, a verification experiment using the optimum medium composition was performed (Table 6). The three replicate experiments yielded an average ethanol concentration of $19.1 \mathrm{~g} / \mathrm{L}$, which was $6.7 \%$ higher than the one obtained in RM medium (17.9 g/L). The good agreement between the predicted and experimental results verified the validity of the model and the existence of an optimal point. The improvement of ethanol production also demonstrated that RSM is a powerful tool to determine the optimal values of the individual factors and the maximum response value.

\subsection{Simplification of the synthetic medium}

To reduce the cost and complexity of the medium, two simplified synthetic media, S1 and S2, were prepared by modifying the composition of the optimum medium obtained by RSM (Table 6). The maximum ethanol production in S1 was $21.9 \mathrm{~g} / \mathrm{L}$, being $14.7 \%$ higher than in the optimum synthetic medium. A similar level of ethanol production (21.7 $\mathrm{g} / \mathrm{L})$ was obtained in S2, which lacked choline chloride, pyridoxine and thiamine. The process ethanol yield in this medium was $73.2 \%$ of the theoretical maximum. Although ethanol production in S2 was slightly lower than in S1, S2 had the advantages of being simpler and cheaper. Therefore, S2 medium was chosen as the final synthetic medium, containing $58.5 \mathrm{~g} / \mathrm{L}$ xylose, $2.65 \mathrm{~g} / \mathrm{L}$ trisodium citrate, $1 \mathrm{~g} / \mathrm{L}$ $\left(\mathrm{NH}_{4}\right)_{2} \mathrm{SO}_{4}, 1 \mathrm{~g} / \mathrm{L} \mathrm{MgSO} \cdot 7 \mathrm{H}_{2} \mathrm{O}, 2 \mathrm{~g} / \mathrm{L} \mathrm{KH}_{2} \mathrm{PO}_{4}$ and 2.74 $\mathrm{mg} / \mathrm{L}$ calcium pantothenate.

\subsection{Ethanol production from glucose and glucose- xylose mixtures}

S3 and S4 media were designed to investigate the growth and ethanol production by recombinant $Z$. mobilis in synthetic media with glucose as the carbon source (Table 6). S3 and S4 had the same composition as S2 except for the carbon source. S3 contained $59 \mathrm{~g} / \mathrm{L}$ glucose, and S4 contained $29.4 \mathrm{~g} / \mathrm{L}$ xylose and $29.7 \mathrm{~g} / \mathrm{L}$ glucose. The final ethanol concentrations in S3 and S4 were 27.3 and $23.6 \mathrm{~g} / \mathrm{L}$, respectively, corresponding to process ethanol yields of $91.1 \%$ and $78.3 \%$, respectively. Although the synthetic medium was optimized with xylose as the carbon source, higher ethanol yields were obtained using glucose or glucose-xylose mixtures. This result indicates that final synthetic media containing glucose or xylose as carbon sources can support the growth and ethanol production by recombinant $Z$. mobilis.

\subsection{Effect of trisodium citrate on ethanol production}

The effect of trisodium citrate on ethanol production was investigated by single-factor experiments. An increasing concentration of trisodium citrate, up to $1.6 \mathrm{~g} / \mathrm{L}$, led to a considerable increase in biomass and ethanol production (Figure 3). However, trisodium citrate levels above $1.6 \mathrm{~g} / \mathrm{L}$ caused a slight decrease in ethanol production, whereas cell 
Table 6 Ethanol production by recombinant Z. mobilis in different media $(n=3)$

\begin{tabular}{|c|c|c|c|c|c|c|}
\hline Components & Optimum synthetic medium & Medium S1 & Medium S2 & Medium S3 & Medium S4 & RM medium \\
\hline Xylose (g/L) & $58.0 \pm 0.36$ & $57.9 \pm 0.41$ & $58.1 \pm 0.29$ & - & $29.4 \pm 0.17$ & $58.2 \pm 0.32$ \\
\hline Glucose (g/L) & - & - & - & $59.0 \pm 0.53$ & $29.7 \pm 0.23$ & - \\
\hline$\left(\mathrm{NH}_{4}\right)_{2} \mathrm{SO}_{4} \quad(\mathrm{~g} / \mathrm{L})$ & 1 & 1 & 1 & 1 & 1 & - \\
\hline $\mathrm{MgSO}_{4} \cdot 7 \mathrm{H}_{2} \mathrm{O}(\mathrm{g} / \mathrm{L})$ & 1 & 1 & 1 & 1 & 1 & - \\
\hline $\mathrm{KH}_{2} \mathrm{PO}_{4}(\mathrm{~g} / \mathrm{L})$ & 2 & 2 & 2 & 2 & 2 & 2 \\
\hline Choline chloride $(\mathrm{mg} / \mathrm{L})$ & 4.35 & 4.35 & - & - & - & - \\
\hline Pyridoxine (mg/L) & 1.81 & 1.81 & - & - & - & - \\
\hline Thiamine (mg/L) & 1.75 & 1.75 & - & - & - & - \\
\hline Methionine (g/L) & 0.1 & - & - & - & - & - \\
\hline Biotin $(\mu \mathrm{g} / \mathrm{L})$ & 33 & - & - & - & - & - \\
\hline Cyanocobalamin $(\mu \mathrm{g} / \mathrm{L})$ & 1 & - & - & - & - & - \\
\hline p-Aminobenzoic acid (mg/L) & 7.64 & - & - & - & - & - \\
\hline Calcium pantothenate $(\mathrm{mg} / \mathrm{L})$ & 2.74 & 2.74 & 2.74 & 2.74 & 2.74 & - \\
\hline Yeast extract (g/L) & - & - & - & - & - & 10 \\
\hline Tetracycline (mg/L) & 15 & 15 & 15 & 15 & 15 & 15 \\
\hline Ethanol (g/L) & $19.1 \pm 0.37$ & $21.9 \pm 0.41$ & $21.7 \pm 0.35$ & $27.4 \pm 0.31$ & $23.6 \pm 0.29$ & $17.9 \pm 0.46$ \\
\hline Metabolic ethanol yield ${ }^{\mathrm{a})} / 0.51^{\mathrm{c})}$ & $80.5 \%$ & $78.6 \%$ & $80.9 \%$ & $91.2 \%$ & $84.2 \%$ & $71.6 \%$ \\
\hline Process ethanol yield ${ }^{\mathrm{b})} / 0.51^{\mathrm{c})}$ & $63.6 \%$ & $74.2 \%$ & $73.2 \%$ & $91.1 \%$ & $78.3 \%$ & $60.3 \%$ \\
\hline
\end{tabular}

a) Metabolic ethanol yield was estimated based on consumed sugar; b) process ethanol yield was estimated based on available sugar; c) a maximum theoretical ethanol yield of $0.51 \mathrm{~g}$ of ethanol per gram of sugar (glucose or xylose) was assumed. "-_indicates that no components are added.

density remained almost unchanged. Slight increases in by-products were observed within the full scope of the study. Biomass and ethanol production were directly proportional to the logarithm of the concentration of trisodium citrate in the range of $0.1-1.6 \mathrm{~g} / \mathrm{L}$.

\section{Discussions}

In this study, statistical experimental designs were used to examine the effects of various components in a synthetic medium on ethanol production, and to develop a model for ethanol production from xylose by recombinant $Z$. mobilis. Among the 19 variables tested by PB experiments, eight components (xylose, $\left(\mathrm{NH}_{4}\right)_{2} \mathrm{SO}_{4}$, trisodium citrate, succinic acid, choline chloride, folic acid, pyridoxine and thiamine) were identified as significant factors for ethanol production. Five of these components (xylose, trisodium citrate, choline chloride, pyridoxine and thiamine) were further optimized by the steepest ascent method. The optimal values of these five components were determined by RSM, and the significant effects of xylose and trisodium citrate were also verified by RSM.

The importance of trisodium citrate for ethanol production was demonstrated. Rising levels of initial citrate in the medium have been reported to lead to an increase in ATP synthesis by substrate-level phosphorylation [21]. The increase

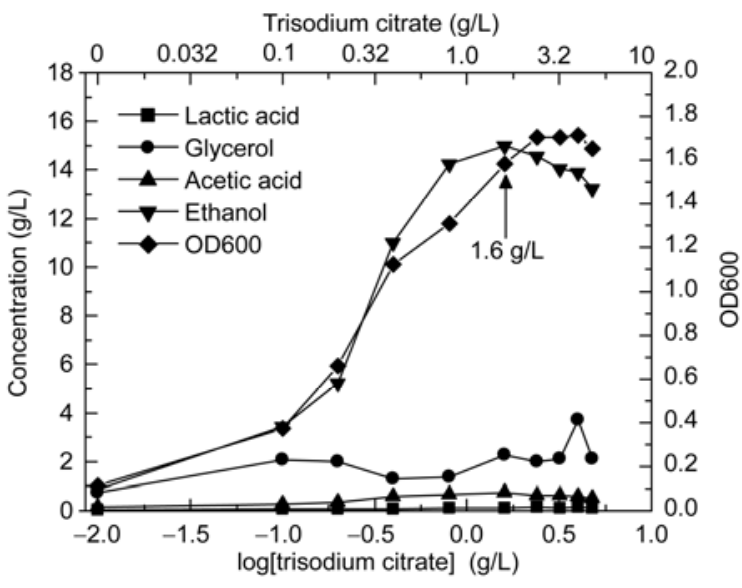

Figure 3 Total growth and product concentration in relation to the logarithm of the concentration of trisodium citrate. Experiments were conducted in duplicates in $250 \mathrm{~mL}$ shake flasks containing $100 \mathrm{~mL}$ of final synthetic medium with different concentrations of trisodium citrate.

in ATP, in turn, promotes higher biomass yields [22,23]. Our results show that elevated levels of trisodium citrate noticeably improve biomass and ethanol production. Product generation in a medium containing citrate generally increases when the formation of by-products is suppressed [12]. Nevertheless, in this study the increase in ethanol production was accompanied by a slight increase in byproducts (Figure 3). Therefore, the enhanced ethanol production in the medium containing trisodium citrate was due, 
to a large extent, to the increased cell density and not to the suppression of by-product formation. Interestingly, biomass and ethanol production were both directly proportional to the logarithm of the concentration of trisodium citrate in the range of $0.1-1.6 \mathrm{~g} / \mathrm{L}$ (Figure 3 ). This changing trend was similar to the growth requirement for pantothenate of wild $Z$. mobilis [24].

Xylose, as the only carbon source in this medium, played a well-known role in cell growth and ethanol production. The optimum xylose concentration was $58.5 \mathrm{~g} / \mathrm{L}$, and a marked inhibition of cell growth was observed when the concentration was above $100 \mathrm{~g} / \mathrm{L}$ (Tables 1 and 3). The engineered strains have been reported to have difficulties dealing with high concentrations of xylose [25]. We thus speculated that the excessive xylose caused great osmotic pressure, metabolic overflow and by-product accumulation, which were disadvantageous to cell growth and product formation [12].

Calcium pantothenate was the only significant factor for ethanol and biomass production identified in the prescreening experiments. Cells did not grow in synthetic media without calcium pantothenate, indicating that pantothenate was the only growth factor of recombinant $Z$. mobilis. This result agrees with previous reports on wild $Z$. mobilis [24]. However, the role of pantothenate in the underlying mechanism promoting growth and ethanol production by $Z$. mobilis is as yet incompletely understood.

The present study identified the key components of a fermentation medium that affect ethanol production, and maximized ethanol production in an optimized medium. The process ethanol yield in the final optimum medium reached $73.2 \%$ of the theoretical yield, which is $36.3 \%$ higher than the ethanol yield obtained in a synthetic xylose-fed $(70 \mathrm{~g} / \mathrm{L})$ continuous culture broth [20]. Interestingly, the two media have very similar compositions, except for 11 extra minor components in the continuous culture broth [20]. Thus, the enhanced ethanol production obtained in the final synthetic medium may be the result of the removal of components which have negative effects on ethanol production, and of the addition of the optimal concentration of each nutrient. The maximum ethanol production in the final synthetic medium increased by $20.7 \%$ compared with the RM medium, in spite of a slight decrease in the rates of growth and substrate consumption. The final synthetic medium composed of simple and cheap inorganic salts is, thus, suitable for growth, ethanol production and metabolism studies of recombinant $Z$. mobilis.

This work was supported by the National Basic Research Program of China (2011CBA00804), the National Natural Science Foundation of China (30900033, 20806055, 20875068) and Project of Introducing Talents of Discipline to Universities (B06006).

1 Bai F W, Anderson W A, Moo-Young M. Ethanol fermentation technologies from sugar and starch feedstocks. Biotechnol Adv, 2008,
26: 89-105

2 Vaheed H, Shojaosadati S, Galip H. Evaluation and optimization of ethanol production from carob pod extract by Zymomonas mobilis using response surface methodology. J Ind Microbiol Biotechnol, 2011, 38: 101-111

3 Rogers P L, Jeon Y J, Lee K J, et al. Zymomonas mobilis for fuel ethanol and higher value products. Adv Biochem Eng Biotechnol, 2007, 108: 263-288

4 Lachke A. Biofuel from D-xylose: The second most abundant sugar. Resonance, 2002, 7: 50-58

5 Zhang M, Eddy C, Deanda K, et al. Metabolic engineering of a pentose metabolism pathway in ethanologenic Zymomonas mobilis. Science, 1995, 267: 240-243

6 Mohagheghi A, Evans K, Chou Y C, et al. Cofermentation of glucose, xylose, and arabinose by genomic DNA-integrated xylose/arabinose fermenting strain of Zymomonas mobilis AX101. Appl Biochem Biotechnol, 2002, 98-100: 885-898

7 Kennedy M, Krouse D. Strategies for improving fermentation medium performance: A review. J Ind Microbiol Biotechnol, 1999, 23 : $456-475$

8 Zhang J, Greasham R. Chemically defined media for commercial fermentations. Appl Microbiol Biotechnol, 1999, 51: 407-421

9 Plackett R L, Burman J P. The design of optimum multifactorial experiments. Biometrika, 1946, 33: 305-325

10 Box G E P, Hunter W G, Hunter J S. Statistics for Experimenters. New York: John Wiley \& Sons, 1978

11 Yatsyshyn V Y, Fedorovych D V, Sibirny A A. Medium optimization for production of flavin mononucleotide by the recombinant strain of the yeast Candida famata using statistical designs. Biochem Eng J, 2010, 49: 52-60

12 Wu Q L, Chen T, Gan Y, et al. Optimization of riboflavin production by recombinant Bacillus subtilis RH44 using statistical designs. Appl Microbiol Biotechnol, 2007, 76: 783-794

13 Abdul Rahman M, Jarmi N, Chaibakhsh N, et al. Modeling and optimization of lipase-catalyzed production of succinic acid ester using central composite design analysis. J Ind Microbiol Biotechnol, 2011, 38: 229-234

$14 \mathrm{Lu} \mathrm{Z}$, He F, Shi Y, et al. Fermentative production of L(+)-lactic acid using hydrolyzed acorn starch, persimmon juice and wheat bran hydrolysate as nutrients. Bioresour Technol, 2010, 101: 3642-3648

15 Maiti B, Rathore A, Srivastava S, et al. Optimization of process parameters for ethanol production from sugar cane molasses by $Z y$ momonas mobilis using response surface methodology and genetic algorithm. Appl Microbiol Biotechnol, 2011, 90: 385-395

16 Sreekumar O, Chand N, Basappa S C. Optimization and interaction of media components in ethanol production using Zymomonas mobilis by response surface methodology. J Biosci Bioeng, 1999, 88: 334-338

17 Karuppaiya M, Sasikumar E, Viruthagiri T, et al. Optimization of process conditions using response surface methodology (RSM) for ethanol production from waste cashew apple juice by Zymomonas mobilis. Chem Eng Commun, 2009, 196: 1425-1435

18 Ma Y Y, Dong H N, Zou S L, et al. Comparison of glucose/xylose co-fermentation by recombinant Zymomonas mobilis under different genetic and environmental conditions. Biotechnol Lett, 2012, 34: 1297-1304

19 De Coninck J, Bouquelet S, Dumortier V, et al. Industrial media and fermentation processes for improved growth and protease production by Tetrahymena thermophila BIII. J Ind Microbiol Biotechnol, 2000, 24: 285-290

20 De Graaf A A, Striegel K, Wittig R M, et al. Metabolic state of Zymomonas mobilis in glucose-, fructose-, and xylose-fed continuous cultures as analysed by ${ }^{13} \mathrm{C}$ - and ${ }^{31} \mathrm{P}-\mathrm{NMR}$ spectroscopy. Arch Microbiol, 1999, 171: 371-385

21 Schmitt P, Diviès C. Effect of varying citrate levels on C4 compound formation and on enzyme levels in Leuconostoc mesenteroides subsp. cremoris grown in continuous culture. Appl Microbiol Biotechnol, 1992, 37: 426-430

22 Salou P, Loubiere P, Pareilleux A. Growth and energetics of Leuco- 
nostoc oenos during cometabolism of glucose with citrate or fructose. Appl Environ Microbiol, 1994, 60: 1459-1466

23 Sáez-Miranda J C, Saliceti-Piazza L, McMillan J D. Measurement and analysis of intracellular ATP levels in metabolically engineered Zymomonas mobilis fermenting glucose and xylose mixtures. Biotechnol Prog, 2006, 22: 359-368
24 Belaich J P, Senez J C. Influence of aeration and of pantothenate on growth yields of Zymomonas mobilis. J Bacteriol, 1965, 89: 11951200

25 Agrawal M, Mao Z, Chen R R. Adaptation yields a highly efficient xylose-fermenting Zymomonas mobilis strain. Biotechnol Bioeng, 2011, 108: 777-785

Open Access This article is distributed under the terms of the Creative Commons Attribution License which permits any use, distribution, and reproduction in any medium, provided the original author(s) and source are credited.

\section{Supporting Information}

Table S1 PB experimental design and ethanol production by recombinant Z. mobilis

The supporting information is available online at csb.scichina.com and www.springerlink.com. The supporting materials are published as submitted, without typesetting or editing. The responsibility for scientific accuracy and content remains entirely with the authors. 